Journal of Mathematics and Informatics

Vol. 10, 2017, 67-74

ISSN: 2349-0632 (P), 2349-0640 (online)

Published 11 December 2017

www.researchmathsci.org

DOI: http://dx.doi.org/10.22457/jmi.v10a9

Journal of

Mathematics and

Informatics

\title{
Observation on the Non-Homogeneous Binary Quadratic Diophantine Equation $5 x^{2}-6 y^{2}=5$
}

\author{
K.Ambika ${ }^{I}$ and T.R.Usha Rani ${ }^{2}$ \\ Department of Mathematics, SIGC, Trichy-620002, Tamilnadu, INDIA. \\ 'e-mail:ambikasri2494@gmail.com \\ 2e-mail:usharanisigc@gmail.com
}

Received 15 November 2017; accepted 6 December 2017

Abstract. Non-homogeneous binary quadratic equation represents hyperbola given by $5 x^{2}-6 y^{2}=5$ is analyzed for its non-zero distinct integer solutions. A few interesting relation between the solution of the given hyperbola, integer solutions for other choices of hyperbola and parabola are obtained.

Keywords: Non-homogeneous quadratic, binary quadratic, integer solutions.

AMS Mathematics Subject Classification (2010): 11D09

\section{Introduction}

The binary quadratic Diophantine equations of the form $a x^{2}-b y^{2}=N,(a, b, N \neq 0)$ are rich in variety and have been analyzed by many mathematicians for their respective integer solutions for particular values of $a, b$ and $N$. In this context, one may refer [113].

This communication concerns with the problem of obtaining non-zero distinct integer solutions to the binary quadratic equation given by $5 x^{2}-6 y^{2}=5$ representing hyperbola. A few interesting relations among its solutions are presented. Knowing an integral solution of the given hyperbola, integer solutions for other choices of hyperbolas and parabolas are presented. Also, employing the solutions of the given equation, special Pythagorean triangle is constructed.

\section{Method of analysis}

The Diophantine equation under consideration is

$$
5 x^{2}-6 y^{2}=5
$$

It is to be noted that (1) represent a hyperbola

Taking $x=X+6 T, y=X+5 T$

In (1), it reduced to the equation

$$
X^{2}=30 T^{2}-5
$$

The smallest positive integer solution $\left(T_{0}, X_{0}\right)$ of (3) is 


\section{K. Ambika and T.R. Usha Rani}

$T_{0}=1, X_{0}=5$

To obtain, the other solutions of (3), consider the pellian equation

$X^{2}=6 T^{2}+1$

whose smallest positive integer solution is

$\widetilde{T}_{0}=2, \tilde{X}_{0}=11$

The general solution $\left(\tilde{T}_{n}, \tilde{X}_{n}\right)$ of (4) is given by

$\tilde{X}_{n}+\sqrt{30} \tilde{T}_{n}=(11+2 \sqrt{30})^{n+1}, n=0,1,2 \ldots$

Since, irrational roots occur in pairs, we have

$$
\tilde{X}_{n}-\sqrt{30} \tilde{T}_{n}=(11-2 \sqrt{30})^{n+1}, n=0,1,2 \ldots
$$

From (5) and (6), solving for $\tilde{X}_{n}, \widetilde{T}_{n}$,we have

$$
\begin{aligned}
& \tilde{X}_{n}=\frac{1}{2}\left[(11+2 \sqrt{30})^{n+1}+(11-2 \sqrt{30})^{n+1}\right]=\frac{1}{2} f_{n} \\
& \tilde{T}_{n}=\frac{1}{2 \sqrt{30}}\left[(11+2 \sqrt{30})^{n+1}-(11-2 \sqrt{30})^{n+1}\right]=\frac{1}{2 \sqrt{30}} g_{n}
\end{aligned}
$$

Applying Brahmagupta lemma between the solutions $\left(T_{0}, X_{0}\right)$ and $\left(\tilde{T}_{n}, \tilde{X}_{n}\right)$, the general solution $\left(T_{n+1}, X_{n+1}\right)$ of (3) is found to be

$T_{n+1}=X_{0} \widetilde{T}_{n}+T_{0} \tilde{X}_{n}$

$X_{n+1}=X_{0} \tilde{X}_{n}+30 T_{0} \tilde{T}_{n}$

$\Rightarrow T_{n+1}=\frac{5}{2 \sqrt{30}} g_{n}+\frac{1}{2} f_{n}$

$X_{n+1}=\frac{5}{2} f_{n}+\frac{\sqrt{30}}{2} g_{n}$

Using (7) and (8) in (2) we have

$$
\begin{aligned}
& x_{n+1}=X_{n+1}+6 T_{n+1}=\frac{11}{2} f_{n}+\sqrt{30} g_{n} \\
& y_{n+1}=X_{n+1}+5 T_{n+1}=5 f_{n}+\frac{55}{2 \sqrt{30}} g_{n}
\end{aligned}
$$

Thus, (9) and (10) represent the integer solutions of the hyperbola (1).

A few numerical examples are given in the following table 1.

Table 1: Examples

\begin{tabular}{|c|c|c|}
\hline$n$ & $x_{n+1}$ & $y_{n+1}$ \\
\hline-1 & 11 & 10 \\
\hline 0 & 241 & 220 \\
\hline 1 & 5291 & 4830 \\
\hline
\end{tabular}


Observation on the Non-Homogeneous Binary Quadratic Diophantine Equation $5 x^{2}-6 y^{2}=5$

\begin{tabular}{|l|l|l|}
\hline 2 & 116161 & 106040 \\
\hline
\end{tabular}

Recurrence relations for $x$ and $\mathrm{y}$ are:

$$
\begin{aligned}
& x_{n+3}-22 x_{n+2}+x_{n+1}=0, n=-1,0,1 \ldots . \\
& y_{n+3}-22 y_{n+2}+y_{n+1}=0, n=-1,0,1 \ldots .
\end{aligned}
$$

\section{A few interesting relations among the solutions are given below}

1. $11 x_{n+2}-x_{n+1}-12 y_{n+2}=0$

2. $11 x_{n+3}-11 x_{n+1}-264 y_{n+2}=0$

3. $11 y_{n+1}-y_{n+2}+10 x_{n+1}=0$

4. $y_{n+2}-y_{n+2}=0$

5. $11 y_{n+3}-10 x_{n+1}-241 y_{n+2}=0$

6. $x_{n+3}-22 x_{n+2}+x_{n+1}=0$

7. $12 y_{n+1}-x_{n+2}+11 x_{n+1}=0$

8. $12 y_{n+3}-241 x_{n+2}+11 x_{n+1}=0$

9. $241 x_{n+1}+264 y_{n+1}-x_{n+3}=0$

10. $x_{n+1}+264 y_{n+3}-241 x_{n+3}=0$

11. $12 y_{n+1}-11 x_{n+3}+241 x_{n+2}=0$

12. $12 y_{n+2}-x_{n+3}+11 x_{n+2}=0$

13. $12 y_{n+3}-11 x_{n+3}+x_{n+2}=0$

14. $11 x_{n+3}-241 x_{n+2}-12 y_{n+1}=0$

15. $y_{n+3}-20 x_{n+2}-y_{n+1}=0$

16. $y_{n+1}-11 y_{n+2}+10 x_{n+2}=0$

17. $y_{n+3}-10 x_{n+2}-11 y_{n+2}=0$

18. $241 y_{n+2}-10 x_{n+3}-11 y_{n+1}=0$

19. $241 y_{n+3}-220 x_{n+3}-y_{n+1}=0$

20. $y_{n+1}+20 x_{n+2}-y_{n+3}=0$

21. $11 y_{n+1}-241 y_{n+2}+10 x_{n+3}=0$

22. $11 y_{n+3}-10 x_{n+3}-y_{n+2}=0$

23. $y_{n+3}-y_{n+3}=0$

24. $10 y_{n+3}-220 y_{n+2}+10 y_{n+1}=0$

25. $220 x_{n+1}-y_{n+3}+241 y_{n+1}=0$ 
K. Ambika and T.R. Usha Rani

\section{Each of the following expressions represents a cubical integer}

i. $\frac{1}{2}\left[\left(88 x_{3 n+3}-4 x_{3 n+4}\right)+3\left(88 x_{n+1}-4 x_{n+2}\right)\right]$

ii. $\frac{1}{44}\left[\left(1932 x_{3 n+3}-4 x_{3 n+5}\right)+3\left(1932 x_{n+1}-4 x_{n+3}\right)\right]$

iii. $\frac{1}{5}\left[\left(110 x_{3 n+3}-120 y_{3 n+3}\right)+3\left(110 x_{n+1}-120 y_{n+1}\right)\right]$

iv. $\frac{1}{55}\left[\left(2410 x_{3 n+3}-120 y_{3 n+4}\right)+3\left(2410 x_{n+1}-120 y_{n+2}\right)\right]$

v. $\frac{1}{1205}\left[\left(52910 x_{3 n+3}-120 y_{3 n+5}\right)+3\left(52910 x_{n+1}-120 y_{n+3}\right)\right]$

vi. $\frac{1}{2}\left[\left(1932 x_{3 n+4}-88 x_{3 n+5}\right)+3\left(1932 x_{n+2}-88 x_{n+3}\right)\right]$

vii. $\frac{1}{55}\left[\left(110 x_{3 n+4}-2640 y_{3 n+3}\right)+3\left(110 x_{n+2}-2640 y_{n+1}\right)\right]$

viii. $\frac{1}{5}\left[\left(2410 x_{3 n+4}-2640 y_{3 n+4}\right)+3\left(2410 x_{n+2}-2640 y_{n+2}\right)\right]$

ix. $\frac{1}{55}\left[\left(52910 x_{3 n+4}-2640 y_{3 n+5}\right)+3\left(52910 x_{n+2}-2640 y_{n+3}\right)\right]$

x. $\frac{1}{1205}\left[\left(110 x_{3 n+5}-57960 y_{3 n+3}\right)+3\left(110 x_{n+3}-57960 y_{n+1}\right)\right]$

xi. $\quad \frac{1}{55}\left[\left(2410 x_{3 n+5}-57960 y_{3 n+4}\right)+3\left(2410 x_{n+3}-57960 y_{n+2}\right)\right]$

xii. $\frac{1}{5}\left[\left(52910 x_{3 n+5}-57960 y_{3 n+5}\right)+3\left(52910 x_{n+3}-57960 y_{n+3}\right)\right]$

xiii. $\frac{1}{50}\left[\left(110 y_{3 n+4}-2410 y_{3 n+3}\right)+3\left(110 y_{n+2}-2410 y_{n+1}\right)\right]$

xiv. $\frac{1}{1100}\left[\left(110 y_{3 n+5}-52910 y_{3 n+3}\right)+3\left(110 y_{n+3}-52910 y_{n+1}\right)\right]$

xv. $\frac{1}{50}\left[\left(2410 y_{3 n+5}-52910 y_{3 n+4}\right)+3\left(2410 y_{n+3}-52910 y_{n+2}\right)\right]$

5. Each of the following expressions represents Nasty number:

i. $\frac{1}{2}\left[24+528 x_{2 n+2}-24 x_{2 n+3}\right]$

ii. $\frac{1}{44}\left[528+11592 x_{2 n+2}-24 x_{2 n+4}\right]$ 
Observation on the Non-Homogeneous Binary Quadratic Diophantine Equation $5 x^{2}-6 y^{2}=5$

iii. $\frac{1}{5}\left[60+660 x_{2 n+2}-720 y_{2 n+2}\right]$

iv. $\frac{1}{55}\left[660+14460 x_{2 n+2}-720 y_{2 n+3}\right]$

v. $\frac{1}{1205}\left[14460+317460 x_{2 n+2}-720 y_{2 n+4}\right]$

vi. $\frac{1}{2}\left[24+11592 x_{2 n+3}-528 x_{2 n+4}\right]$

vii. $\frac{1}{55}\left[660+660 x_{2 n+3}-15840 y_{2 n+2}\right]$

viii. $\frac{1}{5}\left[60+14460 x_{2 n+3}-15840 y_{2 n+3}\right]$

ix. $\frac{1}{55}\left[660+317460 x_{2 n+3}-15840 y_{2 n+4}\right]$

x. $\frac{1}{1205}\left[14460+660 x_{2 n+4}-347760 y_{2 n+2}\right]$

xi. $\quad \frac{1}{55}\left[660+14460 x_{2 n+4}-347760 y_{2 n+3}\right]$

xii. $\quad \frac{1}{5}\left[660+317460 x_{2 n+4}-347760 y_{2 n+4}\right]$

xiii. $\frac{1}{50}\left[660+660 y_{2 n+3}-14460 y_{2 n+2}\right]$

xiv. $\frac{1}{1100}\left[13200+660 y_{2 n+4}-317460 y_{2 n+2}\right]$

\section{Remarkable observations}

6.1. Employing linear combinations among the solutions of (1), one may generate integer solutions for other choices of hyperbola which are presented in table 2 below

Table 2: Hyperbola

\begin{tabular}{|l|l|l|}
\hline $\begin{array}{l}\text { SL.N } \\
\text { o }\end{array}$ & Hyperbola & $\left(X_{n}, Y_{n}\right)$ \\
\hline 1 & $30 X_{n}^{2}-Y_{n}^{2}=480$ & {$\left[\left(88 x_{n+1}-4 x_{n+2}\right),\left(22 x_{n+2}-482 x_{n+1}\right)\right]$} \\
\hline 2 & $30 X_{n}^{2}-Y_{n}^{2}=232320$ & {$\left[\left(1932 x_{n+1}-4 x_{n+3}\right),\left(22 x_{n+3}-10582 x_{n+1}\right)\right]$} \\
\hline 3 & $30 X_{n}^{2}-Y_{n}^{2}=3000$ & {$\left[\left(110 x_{n+1}-120 y_{n+1}\right),\left(660 y_{n+1}-600 x_{n+1}\right)\right]$} \\
\hline
\end{tabular}


K. Ambika and T.R. Usha Rani

\begin{tabular}{|l|l|l|}
\hline 4 & $30 X_{n}^{2}-Y_{n}^{2}=363000$ & {$\left[\left(2410 x_{n+1}-120 y_{n+2}\right),\left(660 y_{n+2}-13200 x_{n+1}\right)\right]$} \\
\hline 5 & $30 X_{n}^{2}-Y_{n}^{2}=17424300$ & {$\left[\left(52910 x_{n+1}-120 y_{n+3}\right),\left(660 y_{n+3}-289800 x_{n+1}\right)\right]$} \\
\hline 6 & $30 X_{n}^{2}-Y_{n}^{2}=480$ & {$\left[\left(1932 x_{n+2}-88 x_{n+3}\right),\left(482 x_{n+3}-10582 x_{n+2}\right)\right]$} \\
\hline 7 & $30 X_{n}^{2}-Y_{n}^{2}=363000$ & {$\left[\left(110 x_{n+2}-2640 y_{n+1}\right),\left(14460 y_{n+1}-600 x_{n+2}\right)\right]$} \\
\hline 8 & $30 X_{n}^{2}-Y_{n}^{2}=3000$ & {$\left[\left(2410 x_{n+2}-2640 y_{n+2}\right),\left(14460 y_{n+2}-13200 x_{n+2}\right)\right]$} \\
\hline 9 & $30 X_{n}^{2}-Y_{n}^{2}=363000$ & {$\left[\left(52910 x_{n+2}-2640 y_{n+3}\right),\left(14460 y_{n+3}-289800 x_{n+2}\right.\right.$} \\
\hline 11 & $30 X_{n}^{2}-Y_{n}^{2}=17424300$ & {$\left[\left(110 x_{n+3}-57960 y_{n+1}\right),\left(317460 y_{n+1}-600 x_{n+3}\right)\right]$} \\
\hline 12 & $30 X_{n}^{2}-Y_{n}^{2}=363000$ & {$\left[\left(2410 x_{n+3}-57960 y_{n+2}\right),\left(317460 y_{n+2}-13200 x_{n+3}\right.\right.$} \\
\hline 13 & $30 X_{n}^{2}-Y_{n}^{2}=300000$ & {$\left[\left(110 y_{n+2}-2410 y_{n+1}\right),\left(13200 y_{n+1}-600 y_{n+2}\right)\right]$} \\
\hline 14 & $30 X_{n}^{2}-Y_{n}^{2}=14520000$ & {$\left[\left(110 y_{n+3}-52910 y_{n+1}\right),\left(289800 y_{n+1}-600 y_{n+3}\right)\right]$} \\
\hline 15 & $30 X_{n}^{2}-Y_{n}^{2}=300000$ & {$\left[\left(2410 y_{n+3}-52910 y_{n+2}\right),\left(289800 y_{n+2}-13200 y_{n+3}\right.\right.$} \\
\hline
\end{tabular}

6.2. Employing linear combination among the solutions for other choices of parabola which are presented in table 3 .

6.3. Consider $p=x+y, q=y$. Observe that $p>q>0$. Treat $p, q$ as the generators of the Pythagorean triangle $T(\alpha, \beta, \gamma)$,

$\alpha=2 p q, \beta=p^{2}-q^{2}, \gamma=p^{2}+q^{2}$.

Then the following interesting relations are observed:
a) $5 \alpha-3 \beta-2 \gamma=-5$
b) $8 \alpha-5 \gamma=12 \frac{A}{P}-5$
c) $\frac{2 A}{P}=x y$ 
Observation on the Non-Homogeneous Binary Quadratic Diophantine Equation $5 x^{2}-6 y^{2}=5$

Table 3: Parabola

\begin{tabular}{|l|l|l|}
\hline SL.NO & Parabola & $\left(X_{n}, Y_{n}\right)$ \\
\hline 1 & $60 X_{n}-Y_{n}^{2}=480$ & {$\left[\left(4+88 x_{2 n+2}-4 x_{2 n+3}\right)_{2}\left(22 x_{n+2}-482 x_{n+1}\right)\right]$} \\
\hline 2 & $1320 X_{n}-Y_{n}^{2}=232320$ & {$\left[\left(88+1932 x_{2 n+2}-4 x_{2 n+4}\right)_{2}\left(22 x_{n+3}-10582 x_{n+1}\right)\right]$} \\
\hline 3 & $150 X_{n}-Y_{n}^{2}=3000$ & {$\left[\left(10+110 x_{2 n+2}-120 y_{2 n+2}\right)_{2}\left(660 y_{n+1}-600 x_{n+1}\right)\right]$} \\
\hline 4 & $330 X_{n}-Y_{n}^{2}=363000$ & {$\left[\left(110+2410 x_{2 n+2}-120 y_{2 n+3}\right)_{2}\left(660 y_{n+2}-13200 x_{n+1}\right)\right]$} \\
\hline 5 & $36150 X_{n}-Y_{n}^{2}=174243000$ & {$\left[\left(2410+52910 x_{2 n+2}-120 y_{2 n+4}\right)_{2}\left(660 y_{n+3}-289800 x_{n+1}\right)\right]$} \\
\hline 6 & $60 X_{n}-Y_{n}^{2}=480$ & {$\left[\left(4+1932 x_{2 n+3}-88 x_{2 n+4}\right)_{2}\left(482 x_{n+3}-10582 x_{n+2}\right)\right]$} \\
\hline 8 & $1650 X_{n}-Y_{n}^{2}=363000$ & {$\left[\left(110+110 x_{2 n+3}-2640 y_{2 n+2}\right)_{2}\left(14460 y_{n+1}-600 x_{n+2}\right)\right]$} \\
\hline 10 & $30 X_{n}-Y_{n}^{2}=3000$ & {$\left[\left(10+2410 x_{2 n+3}-2640 y_{2 n+3}\right)_{2}\left(14460 y_{n+2}-13200 x_{n+2}\right)\right]$} \\
\hline 11 & $1650 X_{n}-Y_{n}^{2}=363000$ & {$\left[\left(110+52910 x_{2 n+3}-2640 y_{2 n+4}\right)_{2}\left(14460 y_{n+3}-289800 x_{n+2}\right)\right]$} \\
\hline 12 & $1650 X_{n}-Y_{n}^{2}=363000$ & {$\left[\left(110+2410 x_{2 n+4}-57960 y_{2 n+3}\right)_{2},\left(317460 y_{n+2}-13200 x_{n+3}\right)\right]$} \\
\hline 13 & $150 X_{n}-Y_{n}^{2}=3000$ & {$\left[\left(10+52910 x_{2 n+4}-57960 y_{2 n+4}\right)_{2}\left(317460 y_{n+3}-289800 x_{n+3}\right)\right]$} \\
\hline 14 & $33000 X_{n}-Y_{n}^{2}=145200000$ & {$\left[\left(2200+110 y_{2 n+4}-52910 y_{2 n+2}\right)_{2}\left(289800 y_{n+1}-600 y_{n+3}\right)\right]$} \\
\hline 15 & $1500 X_{n}-Y_{n}^{2}=300000$ & {$\left[\left(100+2410 y_{2 n+4}-52910 y_{2 n+3}\right),\left(289800 y_{n+2}-13200 y_{n+3}\right)\right]$} \\
\hline
\end{tabular}

\section{Conclusion}

In this paper, we have presented infinitely many integer solutions for the Diophantine equation, represented by hyperbola is given by $5 x^{2}-6 y^{2}=5$. As the binary quadratic Diophantine equations are rich in variety, one may search for the other choices of equations and determine their integer solutions along with suitable properties.

\section{REFERENCES}

1. R.D. Carmichael, Theory of Numbers and Diophantine Analysis, Dover Publications, New York (1950).

2. L.E. Disckson, History of theory of Numbers, Vol.II, Chelsea Publishing co., New York (1952).

3. L.J. Mordell, Diophantine Equations, Academic Press, London (1969).

4. M.A. Gopalan and R. Anbuselvi, Integral solutions of $4 a y^{2}-(a-1) x^{2}=3 a+1$ Acta Ciencia Indica, XXXIV(1) (2008) 291-295.

5. M.A. Gopalan,etal., Integral points on the hyperbola $(a+2) x^{2}-a y^{2}=4 a(k-1)+2 k^{2}, a, k>0$, Indian Journal of Science, 1(2) (2012) 125-126.

6. M.A. Gopalan, S. Devibala and R.Vidhyalakshmi, Integral points on the hyperbola $2 X^{2}-3 Y^{2}=5$, American Journal of Applied Mathematics and Mathematical Sciences, 1 (2012) 1-4. 


\section{K. Ambika and T.R. Usha Rani}

7. S. Vidhyalakshmi,et al., Observations on the hyperbola $a x^{2}-(a+1) y 2=3 a-1$, Discovery, 4(10) (2013) 22-24.

8. K. Meena, M.A. Gopalan and S. Nandhini, On the binary quadratic Diophantine equation $y^{2}=68 x^{2}+13$, International Journal of Advanced Education and Research, 2 (2017) 59-63.

9. K. Meena, S. Vidhyalakshmi and R.Sobana Devi, On the binary quadratic equation $y^{2}=7 x^{2}+32$, International Journal of Advanced Science and Research, 2 (2017) 18-22.

10. K. Meena, MA. Gopalan, S. Hemalatha, On the hyperbola $y^{2}=8 x^{2}+16$, National Journal of Multidisciplinary Research and Developement, 2 (2017) 1-5 .

11. M.A.Gopalan, K.K.Viswanathan and G.Ramya, On the positive Pell equation $y^{2}=12 x^{2}+13$, International Journal of Advanced Education and Research, 2 (2017) 4-8.

12. K.Meena, M.A.Gopalan and V.Sivaranjani, On the positive Pell equation $y^{2}=102 x^{2}+33$, International Journal of Advanced Education and Research, 2 (1) (2017) 91-96.

13. K.Meena, S.Vidhyalakshmi and N. Bhuvaneswari, On the binary quadratic Diophantine equation $y^{2}=10 x^{2}+24$, International Journal of Multidisciplinary Education and Research, 2 (2017) 34-39.

14. Gopalan, et al., Integral points on the hyperbola $(a+2) x^{2}-a y^{2}=4 a(k-1)+2 k^{2}, a, k>0$, Indian Journal of Science, 1(2) (2012) 125-126.

15. M.A.Gopalan and V.Geetha, Observations on some special pellian equations, Cayley J.Math., 2(2) (2013) 109-118.

16. M.A.Gopalan, S.Vidhyalakshmi and A.Kavitha, On the integer solutions of binary quadratic equation, $x^{2}=4\left(k^{2}+1\right) y^{2}+4^{t}, k, t \geq 0$, BOMSR, 2 (2014) 42-46. 